administration, and scoring process, (3) highlight lessons learned and strategies for practical application.

Methods Iterative, participatory action methods guided CTM development and use. The 40-item CTM captures three components of community transformation: Improvement, relationships, and equity. Eighteen coalitions used the CTM at four time-points, selecting areas for improvement deemed salient to their context. Each created action plans to address these areas. Ten semi-structured interviews assessed CTM use and contextual validity.

Results Coalition's CTM scores were averaged across three community transformation dimensions. This revealed wide variation in scores with context-specific strengths and weaknesses; with the exception of one outlier, no coalitions displayed consistent strengths across dimensions. Interviews revealed the CTM used as intended: collaboratively, inclusively, and for strategizing improvements. Users perceived the CTM's greatest value as a needs assessment that operationalized systemic change concepts into descriptive indicators.

Conclusions The CTM is an actionable collaboration tool for coalitions that has recently been adapted into a validated selfassessment for communities to understand improvements in health and equity. As the first translation of maturity models for coalition use, the CTM represents a promising structure for user-led, community-level planning.

\section{OVERCOMING STRUCTURAL INEQUITY IN HEALTH CARE CONTEXTS FOR PEOPLE LIVING WITH MENTAL ILLNESS AND SUBSTANCE USE ISSUES}

Thomas Ungar. Mental Health Commission of Canada, Canada

\subsection{6/bmjoq-2020-IHI.15}

Background Structural stigma arbitrarily limits opportunities and/or constrains the rights of people living with substance use issues and mental illnesses. It is difficult to see -because structural stigma, inequity, and systemic bias 'hides in plain sight,' and because we are just starting to develop measures and undertake research needed to bring structural stigma properly out into the open.

Objectives This presentation will describe the multiple layers of stigma as an inequity impacting quality care for people living with mental illness and substance use issues. It will also identify opportunities for interventions to improve and dismantle structural inequities within health-care organizations summarized in our framework to action.

Methods To better understand its effects and impacts, the Mental Health Commission of Canada Research Team conducted a comprehensive literature review, qualitative research among people with lived experience, and a promising partners report to identify key priorities and areas of focus for structural change in the health-care system.

Results Early findings demonstrate that addressing structural stigma in health-care contexts requires a multipronged approach, working towards cultural change by improving attitudes and practices of health-care practitioners, strengthening integration and coordination of care, using a stigma-informed lens to evaluate and revise policies and practices, prioritizing the meaningful inclusion of people with lived and living experience at all levels, especially within research, policy and

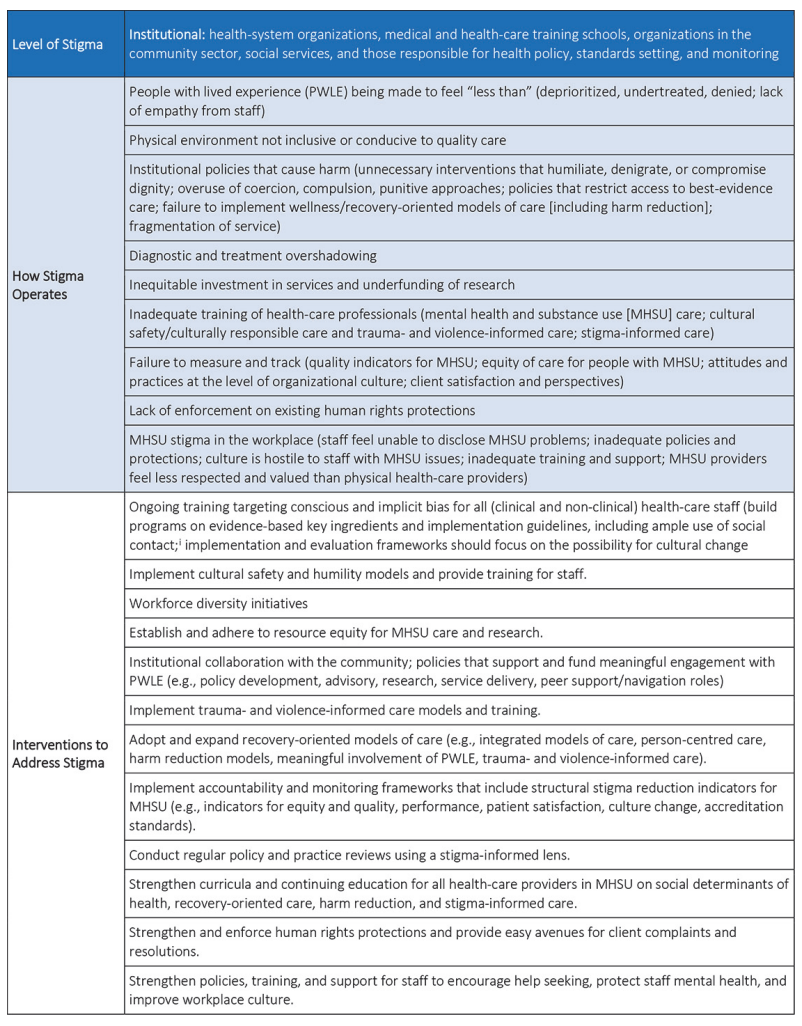

Abstract 15 Figure 1 Combating mental illness- and substance userelated structural stigma in healthcare: A framework for action

\begin{tabular}{|c|c|}
\hline Level of Stigma & $\begin{array}{l}\text { Institutional: health-yystem organizations, medical and health-care training schools, organizations in the } \\
\text { community sector, social services, and those responsible for health policy, standards setting, and monitoring }\end{array}$ \\
\hline \multirow{12}{*}{$\begin{array}{l}\text { Potential } \\
\text { Outcomes }\end{array}$} & An institutional environment that is inclusive, welcoming, diverse, and safe \\
\hline & Organizations that can meet the needs of all populations, including PWLE \\
\hline & A reduction in stigmatizing beliefs and attitudes among staff and across the organization \\
\hline & Improved patient/client ratings of care, satisfaction, and trust \\
\hline & Improved patient/client outcomes (physical and mental health for PWLE; quality of life for PWLE) \\
\hline & Earlier engagement in care for PWLE due to earlier help seeking \\
\hline & Better retention in care and treatment for PWLE \\
\hline & More appropriate and best-evidence care provided to PWLE \\
\hline & Greater compassion satisfaction among staff \\
\hline & Improved mental health of health-care staff \\
\hline & Less time off work; improved worker retention \\
\hline & MHSU providers that feel valued and equitably compensated within the health-care system \\
\hline
\end{tabular}

Abstract 15 Figure 2 Adapted from "Table 2 - Action Framework for Building an Inclusive Health System," by the Public Health Agency of Canada, Addressing stigma: Towards a more inclusive health system, The chief public health officer's report on the state of public health in Canada (p. 41), 2019, Ottawa, Canada: Copyright 2019 by Her Majesty the Queen in Right of Canada, as represented by the Minister of Health

service delivery, and establishing mechanisms to monitor structural stigma (figures 1 and 2).

Conclusions This research brought into focus a comprehensive picture of the problem of structural stigma inequity; how it is experienced, how it impacts on health and quality of life outcomes, and important strategies and approaches for reshaping the way health service delivery and care is provided for people with substance use and mental health problems. These include sharing innovative models of care, developing training modules, and developing measures to identify structural stigma. 